\title{
Bone-Subtracted Spinal CT Angiography Using Nonrigid Registration for Better Visualization of Arterial Feeders in Spinal Arteriovenous Fistulas
}

(D). Nishii, A.K. Kono, M. Nishio, N. Negi, A. Fujita, E. Kohmura, and K. Sugimura

\begin{abstract}
BACKGROUND AND PURPOSE: Pretreatment diagnosis for the location of shunts and arterial feeders of spinal arteriovenous fistulas is crucial. This study aimed to evaluate the utility of subtracted CT angiography imaging by using nonrigid registration (R-CTA) in patients with spinal arteriovenous fistulas compared with conventional CTA imaging.
\end{abstract}

MATERIALS AND METHODS: The records of 15 consecutive subjects (mean age, 65 years; 2 women) who had undergone CTA and digital subtraction angiography for clinically suspected spinal arteriovenous fistula were reviewed. From CTA images obtained at the arterial and late arterial phases, warped images of the late arterial phase were obtained by using nonrigid registration that was adjusted to the arterial phase images. R-CTA images were then obtained by subtracting the warped images from the arterial phase images. The accuracies of using nonrigid registration and conventional spinal CTA and the time required for detecting arterial feeders in spinal arteriovenous fistulas were analyzed for each patient with DSA results as a standard reference. The difference between R-CTA and conventional spinal CTA was assessed by the Welch test and the McNemar $\chi^{2}$ test.

RESULTS: R-CTA had a higher accuracy compared with conventional spinal CTA (80\% versus $47 \%, P=.025)$. The time for interpretation was reduced in R-CTA compared with conventional spinal CTA (45.1 versus 97.1 seconds, $P=.002$ ).

CONCLUSIONS: Our subtracted CTA imaging by using nonrigid registration detects feeders of spinal arteriovenous fistulas more accurately and quickly than conventional CTA.

ABBREVIATIONS: ANTs = Advanced Normalization Tools software; C-CTA = conventional spinal CTA; R-CTA = bone-subtracted spinal CTA using novel nonrigid registration; SAVF = spinal arteriovenous fistula; $\mathrm{SyN}=$ symmetric diffeomorphic image normalization algorithm

S pinal arteriovenous fistulas (SAVFs) are the most common spinal vascular malformation. ${ }^{1}$ Early and accurate diagnosis with proper treatment is required for avoiding progressive spinal cord symptoms. ${ }^{2}$ Treatment in SAVFs is achieved by shunt occlusion with an endovascular or surgical approach. Pretreatment diagnosis for the location of shunts and arterial feeders of SAVFs is crucial. ${ }^{1}$ Selective spinal digital subtraction angiography is a definitive examination in the diagnosis and planning of the treatment of SAVFs. However, selective catheterization for each segmental artery is relatively invasive and time-consuming and requires expertise. ${ }^{3}$

Received January 6, 2015; accepted after revision March 29.

From the Departments of Radiology (T.N., A.K.K., M.N., K.S.) and Neurosurgery (A.F., E.K.), Kobe University Graduate School of Medicine, Kobe, Japan; and Division of Radiology (N.N.), Center for Radiology and Radiation Oncology, Kobe University Hospital, Kobe, Japan.

Please address correspondence to Tatsuya Nishii, MD, Department of Radiology, Kobe University Graduate School of Medicine, 7-5-1 Kusunoki-cho, Chuo-ku, Kobe, Hyogo 650-0017 Japan; e-mail: ttsynishii@gmail.com

http://dx.doi.org/10.3174/ajnr.A4435
Therefore, spinal CT angiography has been introduced to detect the location of shunts and feeders as a noninvasive imaging method. ${ }^{3-6}$ Whether spinal CTA can be substituted for the invasive method of spinal DSA is still controversial. However, preangiographic detection of the location of feeders makes it possible to avoid the invasive method of DSA due to reduction in fluoroscopy time. ${ }^{7}$ Spinal CTA has been widely used because of its simplicity in the clinical setting, after introduction for visualization of the artery of Adamkiewicz. ${ }^{8,9}$ However, CTA has several issues that need to be considered as follows: 1) The detectability of arterial feeders is insufficient, ${ }^{5,6}$ 2) the reproducibility is relatively lower than that of magnetic resonance angiography, ${ }^{6}$ and 3 ) identifying these feeders is sometimes time-consuming. These problems are because the feeders are small and run close to the osseous structures, which show high attenuation in CT images and cause artifacts, such as blooming and streak artifacts, to the neighboring area.

Subtracted CTA is one of the solutions for distinguishing the osseous structures and enhanced vessels. ${ }^{10}$ If the bones can be removed from spinal CTA, the diagnosis of feeders to the SAVF 


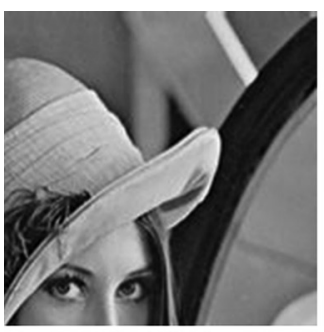

A. original image

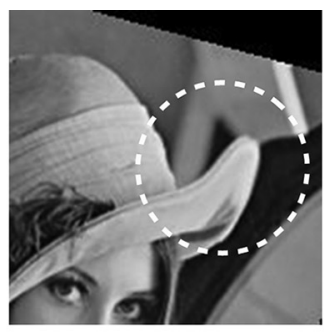

B. deformed image

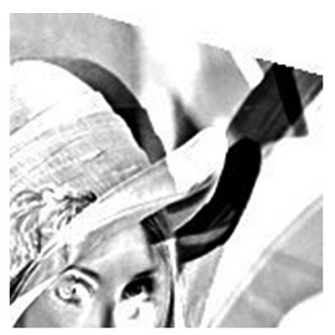

C. subtraction

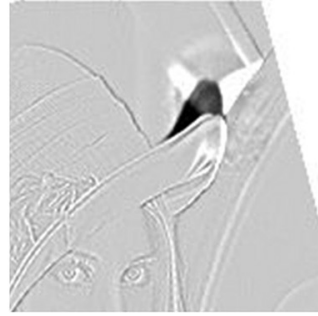

D. subtraction (rigid)

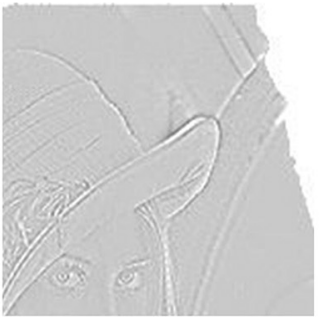

E. subtraction (non-rigid)

FIG 1. A, An original image. $B$, Image generated by rotating $A$ and deforming the tip of the hat (dashed circle). $C$, An ordinary subtracted image that was obtained by subtracting image $B$ from $A$. This step causes misregistration in which a large amount of the white or black area is seen. $D$ and $E$, Subtracted images that were obtained by subtracting image $A$ from the warped image of image $B$ created by rigid and nonrigid registration, respectively. $D$, Deformation of the hat remains and leads to misregistration around the tip of the hat. E, Perfect subtraction by restoring the rotation and deformation.

will be improved. Recently, bone-subtraction CTA has been widely used in intracranial lesions. ${ }^{11-13}$ However, the application of the subtraction technique to spinal lesions is still challenging. To the best of our knowledge, this application has not been reported because the patient's breathing and involuntary motions cause the body position to easily change between the 2 datasets. In most parts of the body, except for cranial lesions, deformation and distortion between 2 datasets will occur. ${ }^{14}$ Misregistration is inevitable when the conventional subtraction method is used. To overcome this misregistration, we introduced rigid or nonrigid registration (Fig 1). Rigid registration is a technique in which 1 image is subtracted with parallel shift or rotation. An example of the application of this method is brain perfusion imaging. Moreover, compared with rigid registration, nonrigid registration is a better processing method, compensating for organ motion or transformation between 2 datasets, ${ }^{14}$ and this can minimize misregistration, even if the target organ moves between the acquisition of images. Recently, several novel nonrigid registration algorithms were introduced, and the performance of these methods is improving. ${ }^{15,16}$

We hypothesized that subtracted spinal CTA imaging by using nonrigid registration (R-CTA) provides precise subtraction and facilitates the diagnosis of arterial feeders to SAVFs. This study aimed to evaluate the utility of R-CTA in patients with SAVFs compared with conventional CTA imaging (C-CTA).

\section{MATERIALS AND METHODS}

Our institutional review board approved this retrospective study. Written informed consent from all subjects was waived by the institutional review board because of the retrospective nature of the study. The patient records and information were anonymized and de-identified before analysis.

\section{Subjects}

The records of 15 consecutive subjects (mean age, 65 years; range, 39-87 years; 2 females) who had undergone CTA and DSA for clinically suspected SAVFs between June 2009 and December 2013 were retrospectively reviewed.

\section{DSA Technique}

Selective spinal DSA was performed by a trained board-certified neurosurgeon (A.F., with 20 years' experience) in a biplane angiography suite (Artis zee BA twin; Siemens, Erlangen, Ger- many) by injecting $3 \mathrm{~mL}(1 \mathrm{~mL} / \mathrm{s})$ of $300-\mathrm{mg} / \mathrm{mL}$ iodinated contrast agent (iopamidol, Oypalomin 300; Fuji Pharma, Tokyo, Japan; or iopromide, Proscope 300; Alfresa Pharma, Osaka, Japan) into the segmental arteries. The images were obtained with the following parameters: 4 frames/s, $720 \times 720$ matrix, and 32-cm FOV. When prior CTA findings suggested the location of the feeders, the predicted level was first selected. After the main feeder was recognized, the bilateral segmental arteries ranging from 3 levels above to 3 levels below the identified feeders were assessed to identify the collateral feeders for complete evaluation. If the feeders were not identified, all the bilateral segmental arteries from the cranial level to the median sacral arteries, the bilateral subclavian arteries, and the bilateral iliac arteries were assessed. The DSA diagnosis was performed by the same neurosurgeon.

\section{Acquisition and Reconstruction of CTA}

All the CTA examinations were performed with a 64-detector row CT scanner (Aquilion 64; Toshiba Medical Systems, Tokyo, Japan) by using the method for visualization of the artery of Adamkiewicz (as previously described). ${ }^{17}$ The parameters were set as follows: 0.5 -mm collimated section width, 0.60 seconds per rotation, 0.641 pitch, $120 \mathrm{kV}$, and $400 \mathrm{mAs}$. Iopamidol at $370 \mathrm{mg} \mathrm{I} / \mathrm{mL}$ (Iopamiron 370; Bayer Yakuhin, Osaka, Japan) was injected via a 20 -ga catheter in the right antecubital vein $(100 \mathrm{~mL}, 5 \mathrm{~mL} / \mathrm{s})$, followed by a $30-\mathrm{mL}$ saline flush. Bolus tracking was used with an ROI at the descending aorta. The scan was automatically started 7 seconds after contrast enhancement of the ROIs reached a threshold of $150 \mathrm{HU}$. The scan covered the entire spinal canal from the foramen magnum to the coccygeal bone. To avoid misreading between the radiculomedullary arteries and veins, we consecutively repeated dual-phase dynamic scanning to obtain images in the arterial and late arterial phases. The interval between the phases was approximately 40 seconds, which was needed to perform the arterial phase scanning and to move the table into the starting position for the late arterial phase scan. This interval differed slightly on the basis of the patient's body size. The CT dose index of the arterial and late arterial phases was set as $47.1 \mathrm{mGy}$ for each phase.

The data were reconstructed in the axial plane with a $0.5-\mathrm{mm}$ section thickness, $0.5-\mathrm{mm}$ reconstruction interval, 200-mm FOV, and a medium soft-tissue convolution kernel.

AJNR Am J Neuroradiol 36:2400-06 Dec 2015 www.ajnr.org 


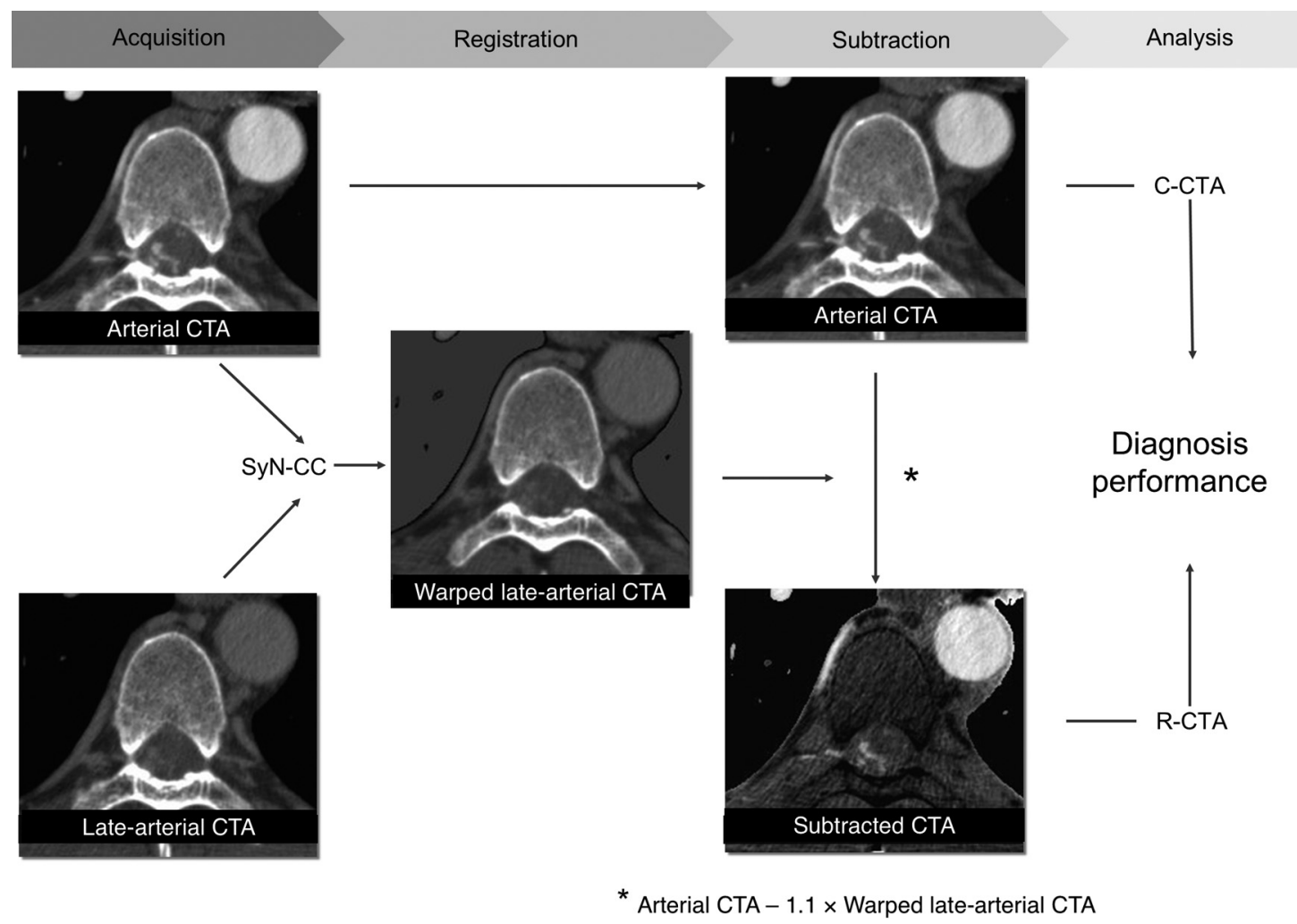

FIG 2. First, warped images of the late arterial phase were obtained by using nonrigid registration (SyN-cross-correlation) adjusted to the arterial phase images. The subtracted spinal CTA by using nonrigid registration was then obtained by subtracting warped images that were multiplied by 1.1 from the arterial phase images. R-CTA and conventional spinal CTA were analyzed with digital subtraction angiography results as a reference standard, and the difference in diagnostic performance was assessed.

\section{Postprocessing Method}

One author (T.N., board-certified diagnostic radiologist with 6 years' experience) who was blinded to the subjects' identities performed further image postprocessing. The schema of the postprocessing methods is shown in Fig 2.

Each dataset was cropped at the center of the image with $256 \times$ 256 pixels, and the Otsu segmentation method was used to remove the lungs with ImageJ software (National Institutes of Health, Bethesda, Maryland). Warped images of the late arterial phase were obtained by using nonrigid registration adjusted to the arterial phase images. Nonrigid registration was performed by using open-source Advanced Normalization Tools software (ANTs; http://stnava.github.io/ANTs/). Details of this process are explained in the next paragraph. R-CTA images were then obtained by subtracting the warped images from the arterial phase images. Weighted subtraction images were used to invert bone attenuation and to easily recognize the level of the intervertebral foramen. Weighted subtraction images were obtained by subtracting warped images that were multiplied by 1.1 from the arterial phase images.

\section{Symmetric Diffeomorphic Image Normalization Algorithm}

The symmetric diffeomorphic image normalization algorithm (SyN), ${ }^{15}$ which is provided through ANTs, is a nonrigid registration algorithm that performs well. ${ }^{16}$ SyN uses an optimization strategy based on minimizing the shape and appearance distances between the input data and reference data. Furthermore, cross- correlation, a similarity metric that is commonly used for intramodality registration, was specified and included when performing the SyN.

\section{Analyses of Images}

Two board-certified diagnostic radiologists (T.N., 6 years' experience; A.K.K., 11 years' experience) who were blinded to the identity of subjects independently performed the following analyses by using OsiriX Imaging Software (http://www.osirixviewer.com). They were allowed to change the window level or width and the image size as common practice. In addition to the axial images, multiplanar reformation images and thin-slab maximum-intensity-projection images were used.

For the preliminary analysis, the initial 5 cases of R-CTA were compared with the bone-subtracted image with rigid registration by the 5 -point scoring system for subtraction performance $(5=$ excellent, $4=$ acceptable, $3=$ intermediate, $2=$ partial, and $1=$ inadequate). The rigid registration was performed by using ANTs with the same datasets used for R-CTA.

Subject-based and intervertebral foramen-based analyses were performed separately in at least 2-week intervals. First, for subject-based analysis, the levels of feeders to the SAVF were recorded, and these were identified from the feeders' continuity between the aorta and abnormal spinal vessels. Furthermore, the required time to interpret the images was concurrently recorded. The 2 examiners performed this analysis independently.

Second, for the intervertebral foramen-based analysis, the in- 
Table 1: Subject characteristics and the level of feeders as shown by spinal digital subtraction angiography results

\begin{tabular}{|c|c|c|c|c|c|c|}
\hline \multirow[b]{2}{*}{ Case } & \multirow[b]{2}{*}{ Age (yr) } & \multirow[b]{2}{*}{ Sex } & \multirow{2}{*}{$\begin{array}{c}\text { Clinical } \\
\text { Manifestation }\end{array}$} & \multicolumn{2}{|c|}{ Feeder } & \multirow[b]{2}{*}{ Type } \\
\hline & & & & Side and Level & Origin & \\
\hline 1 & 60 & $\mathrm{~F}$ & Paraplegia & - & - & No AVF \\
\hline 2 & 83 & $M$ & Claudication & Lt. L1 & LA & $\mathrm{D}$ \\
\hline 3 & 71 & $M$ & Paraplegia & Rt. L2 & LA & D \\
\hline 4 & 78 & $M$ & Paraplegia & Rt. T7, Lt. T11 & ICA & $\mathrm{D}$ \\
\hline 5 & 53 & $M$ & Paraplegia & Rt. T9 & ICA & $D$ \\
\hline 6 & 74 & $M$ & Paraparesis & - & - & No AVF \\
\hline 7 & 49 & $M$ & Paraplegia & Lt. T6 & ICA & PM \\
\hline 8 & 66 & $M$ & Paraplegia & Lt. T6, Lt. T7 & ICA & D \\
\hline 9 & 39 & $M$ & Paraplegia & Lt.T5, Lt. T6 & ICA & D \\
\hline 10 & 57 & $M$ & Paraplegia & Rt. L2 & LA & D \\
\hline 11 & 67 & $\mathrm{~F}$ & Paraplegia & Lt. L3 & LA & PM \\
\hline 12 & 80 & $M$ & Paraplegia & Rt. T4, Rt. T5 & ICA & D \\
\hline 13 & 45 & $M$ & Paraplegia & Rt. T4 & ICA & D \\
\hline 14 & 71 & $M$ & Paraplegia & Lt. T3, Rt. T5 & ICA & PM \\
\hline 15 & 87 & $M$ & Paraplegia & Lt. T4 & ICA & D \\
\hline
\end{tabular}

Note:-D indicates dural AVF; LA, lumber artery; PM, perimedullary AVF; -, data not available; ICA, intercostal artery; T, thoracic spine; L, lumbar spine; Rt., right; Lt, left
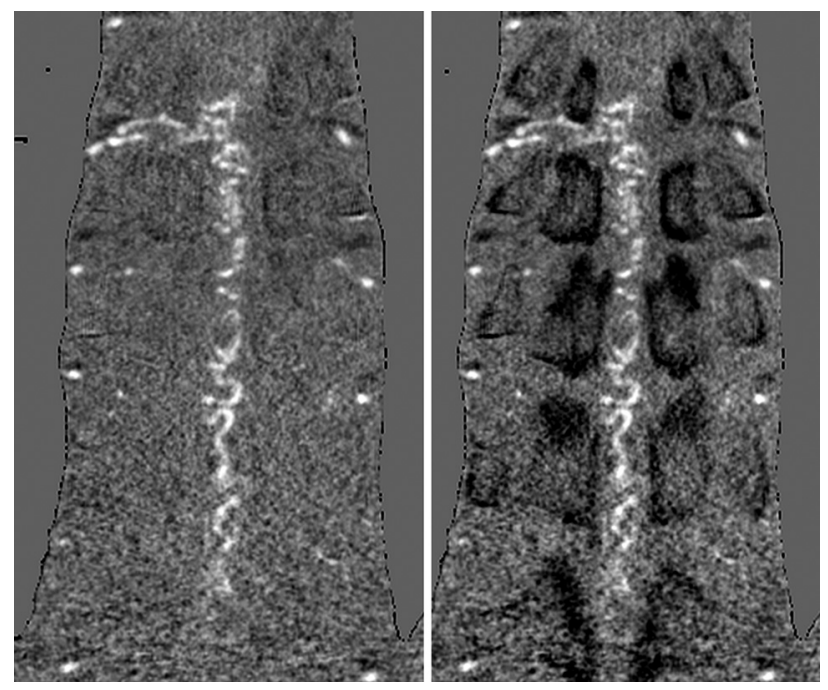

FIG 3. In an ordinary subtraction image (left), determining the level of the vertebra is difficult because the background is totally equaled. However, in a weighted subtraction image (right), the bone is displayed as a darker structure than background attenuation.

tervertebral foramen was assessed from the level of the third thoracic vertebra to the level of the third lumbar vertebra (390 foramens). The diagnostic likelihood of the presence of a feeder was scored on a 5 -point scale $(1=$ definitely negative, $5=$ definitely positive) with a consensus reading of the 2 radiologists.

For quantitative image-quality analysis, the SDs of C-CTA and R-CTA were obtained by setting the circular ROI in the descending aorta at the center level of the 10th thoracic vertebra. The image noise was determined as the $\mathrm{SD}$ of the $\mathrm{CT}$ value in the descending aorta.

\section{Statistical Analyses}

For assessment of diagnostic performance, the result of DSA was used as the reference standard.

For the preliminary analysis, the scores of subtraction performance of C-CTA and R-CTA were compared using the CochranArmitage test.

For subject-based analysis, only when the results of each CTA and DSA were perfectly matched did we judge the diagnosis accurate. The differences in the accuracy and time to interpretation between C-CTA and R-CTA were assessed by the McNemar $\chi^{2}$ test and the Welch test, respectively.

For intervertebral foramen-based analysis, if the score was $\geq 4$, we first determined that a feeder was present at the vertebral foramen. Second, the generalized estimation equation was used to generate a model to marginalize the intersubject effect. The sensitivity, specificity, and accuracy for C-CTA and RCTA were then calculated, and the differences between C-CTA and R-CTA were assessed by each odds ratio estimated by using the generalized estimation equation. The link function was set as a logit link, and an independent working correlation matrix was used for the generalized estimation equation.

For quantitative image analysis, the image noises of C-CTA and R-CTA were compared using the Welch test.

For statistical analysis, JMP 9.0 (SAS Institute, Cary, North Carolina) and R statistical computing software (http://www.rproject.org) were used. The significance level was set at $P=.05$.

\section{RESULTS}

CTA and selected spinal DSA were successfully performed in all 15 subjects. A summary of the characteristics of the subjects and the level of feeders from the results of DSA are shown in Table 1. From the preliminary analysis, R-CTA showed significantly higher subtraction performance than subtraction with the rigid registration (median score, 5 versus 2 , respectively; $P=.001$ ). Thus, for bone subtraction in spinal CTA, the use of nonrigid registration was considered more suitable than the use of rigid registration.

Illustrative cases of a 53-year-old man (case 5) and 57-year-old man (case 10) are shown in Figs 4 and 5, respectively. In case 5, although the feeder from the right ninth intercostal artery (Fig $4 A$ ) was detected by using C-CTA (Fig $4 B$ ) and R-CTA (Fig 4C), the continuity of the feeder and the aorta was clear when R-CTA was used. In case 10, by using C-CTA (Fig 5B), a false feeder from the right 12th intercostal artery was only observed instead of the true feeder from the right second lumbar artery (Fig $5 A$ ), while this true feeder was clearly visualized by using R-CTA (Fig 5C).

In subject-based analysis, the accuracies of C-CTA compared with R-CTA were $47 \%$ (7/15) versus $80 \%(12 / 15)$ by observer 1 and $40 \%(6 / 15)$ versus $73 \%(11 / 15)$ by observer 2 , respectively. The required time for detecting feeders of C-CTA compared with R-CTA was 97.1 seconds versus 45.1 seconds by observer 1 and 89.5 seconds versus 45.6 seconds by observer 2 , respectively. RCTA was significantly more accurate $(P=.025$ in both observers $)$ and reduced the time for interpretation $(P=.002$ and $P=.020$, respectively) compared with C-CTA (Table 2).

In intervertebral foramen-based analysis, the diagnostic performance of C-CTA compared with R-CTA was as follows: sensi- 

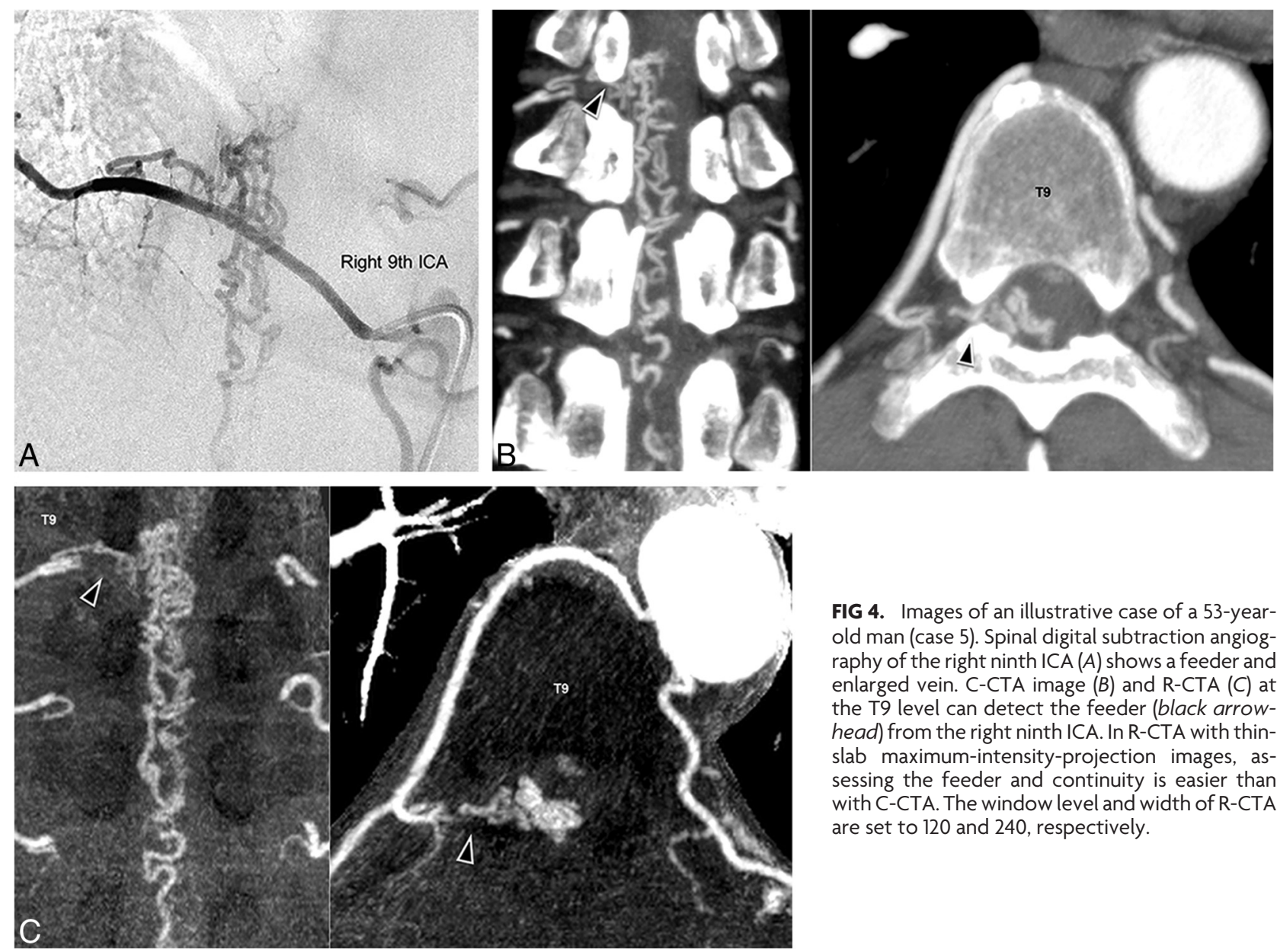

FIG 4. Images of an illustrative case of a 53-yearold man (case 5). Spinal digital subtraction angiography of the right ninth ICA $(A)$ shows a feeder and enlarged vein. C-CTA image $(B)$ and R-CTA $(C)$ at the T9 level can detect the feeder (black arrowhead) from the right ninth ICA. In R-CTA with thinslab maximum-intensity-projection images, assessing the feeder and continuity is easier than with C-CTA. The window level and width of R-CTA are set to 120 and 240 , respectively.

tivity, $44.4 \%$ versus $72.2 \%$; specificity, $98.1 \%$ versus $99.5 \%$; and accuracy, 95.6\% versus 98.2\%, respectively (Table 2). R-CTA showed a significantly higher accuracy (odds ratio $=2.494 ; 95 \%$ confidence interval, 1.037-5.996; $P=.041)$ compared with C-CTA.

The quantitative image-quality analysis showed that the image noise was higher in R-CTA than C-CTA (27.9 \pm 15.5 versus $18.6 \pm 7.0 \mathrm{HU}$, respectively; $P=.047)$.

The radiation exposure by using the dose-length product for dual-phase CTA was $5238.1 \pm 1220.8 \mathrm{mGy} \times \mathrm{cm}$.

\section{DISCUSSION}

Our study showed that R-CTA had a significantly better accuracy than C-CTA, and R-CTA reduced the time required for detection of arterial feeders to the SAVF. Furthermore, in intervertebral foramen-based analysis, R-CTA was significantly more accurate than C-CTA.

The diagnostic accuracy of feeders of SAVFs by using nonsubtracted CTA was previously reported as 58\%-90\%. ${ }^{3,5,6}$ The diagnostic accuracy of C-CTA (40\%-47\%) in our study was relatively low compared with that in previous studies. ${ }^{3,5,6}$ The reason for this difference between studies might be because of a difference in study populations. Thirty-eight percent $(5 / 13)$ of patients had 2 feeders in this study, while the previous studies mentioned only 1 vessel as the main feeder in their populations. Because smaller feeders are easy to miss, the accuracy of C-CTA was not high in this study. Dynamic contrast-enhanced MR angiography is another noninvasive imaging method, which has a detectability of feeders of 40\%-93\%. ${ }^{3,6,7}$ Dynamic contrast-enhanced MR angiography has several advantages, including separating the signal between bone and vessels; and it also performs multiphase scanning without any radiation exposure. The advantages of CTA compared with MR imaging are that it has simple and rapid acquisition, wide coverage, and high versatility. ${ }^{3,6}$ In particular, for surgical planning, 3D visualization of both vessels and bones on CTA images is advantageous. Despite the above-mentioned advantages, radiation exposure is a disadvantage in CTA. However, as the CT machine and reconstruction methods improve, they will be able to reduce radiation exposure.

With R-CTA, separation from bony structures and reduction of blooming artifacts ${ }^{18}$ and extensibility by using thin-slab MIP for easier analysis of continuity ${ }^{19}$ from the aorta to feeders could explain the improving diagnostic performance. Several disadvantages of R-CTA should be considered. First, the subtracted image is theoretically noisier than the original images. ${ }^{19}$ The quantitative image-quality analysis showed that the image noise was higher in R-CTA than in C-CTA. However, the diagnostic performance of R-CTA was not obscured. Thus, the effect of increasing noise was low enough to diagnose the level of feeders in this study because of good visibility of vessels in R-CTA. Second, preparation of 2 CT datasets for obtaining R-CTA could be an issue be- 

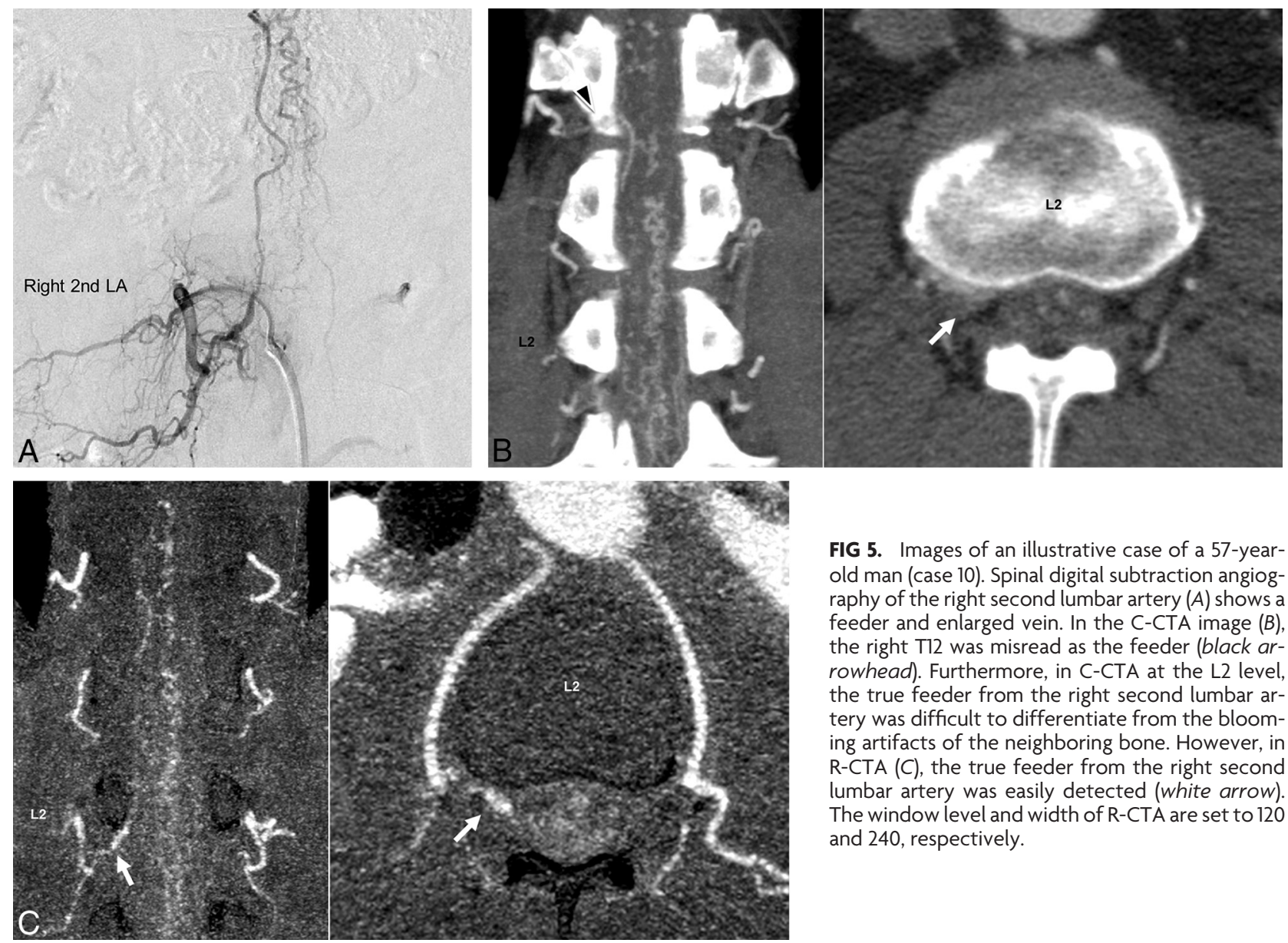

FIG 5. Images of an illustrative case of a 57-yearold man (case 10). Spinal digital subtraction angiography of the right second lumbar artery $(A)$ shows a feeder and enlarged vein. In the C-CTA image $(B)$, the right $\mathrm{T} 12$ was misread as the feeder (black arrowhead). Furthermore, in C-CTA at the L2 level, the true feeder from the right second lumbar artery was difficult to differentiate from the blooming artifacts of the neighboring bone. However, in R-CTA $(C)$, the true feeder from the right second lumbar artery was easily detected (white arrow). The window level and width of R-CTA are set to 120 and 240 , respectively.

Table 2: Differences in diagnostic performance of C-CTA and R-CTA

\begin{tabular}{|c|c|c|c|c|}
\hline Variables & C-CTA & R-CTA & $O^{a}$ & $P$ Values \\
\hline \multicolumn{5}{|l|}{ Subject-based analysis } \\
\hline \multicolumn{5}{|c|}{ Feeder detection accuracy (\%) } \\
\hline Observer 1 & $47(7 / 15)$ & $80(12 / 15)$ & & $.025^{\mathrm{b}}$ \\
\hline Observer 2 & $40(6 / 15)$ & $73(11 / 15)$ & & $.025^{\mathrm{b}}$ \\
\hline \multicolumn{5}{|c|}{ Time required for diagnosis (sec) } \\
\hline Observer 1 & $97.1 \pm 37.4$ & $45.1 \pm 23.6$ & & $.002^{\mathrm{b}}$ \\
\hline Observer 2 & $89.5 \pm 60.0$ & $45.6 \pm 24.1$ & & $.020^{\mathrm{b}}$ \\
\hline \multicolumn{5}{|c|}{ Intervertebral foramen-based analysis } \\
\hline Sensitivity $(\%)^{c}$ & $44.4(22.2-66.7)$ & $72.2(50.0-94.4)$ & $3.26(0.82-13.0)$ & .095 \\
\hline Specificity (\%) ${ }^{c}$ & $98.1(97.8-99.5)$ & $99.5(98.7-100)$ & $3.55(0.87-14.5)$ & .077 \\
\hline Accuracy $(\%)^{c}$ & $95.6(93.8-97.2)$ & $98.2(96.9-99.2)$ & $2.49(1.04-6.00)$ & $.041^{\mathrm{b}}$ \\
\hline
\end{tabular}

${ }^{a}$ ORs of R-CTA against C-CTA are shown.

bSignificant.

c 95\% Confidence intervals are shown.

were needed in each case for registration of the 2 large image datasets ( $>1000 \mathrm{im}$ ages per 1 dataset), even if we used a powerful workstation. For clinical use, the current level of postprocessing time is unacceptable. However, further technical innovations of algorithms and workstations would solve this issue. For instance, the reduction of computation time by $93 \%$ compared with ANTs has been reported by using the deformable registration method in a hybrid framework. ${ }^{21}$ While there are some issues, notably, nonrigid registration was successfully applied to spinal CTA and it could

cause of the radiation dose. High-radiation-dose imaging is usually needed for the detection of feeders of SAVFs. Although dual-phase spinal CTA had been reported beneficial for avoiding contamination between the radiculomedullary arteries and veins, ${ }^{9,17,20}$ its contribution to detecting the feeders of SAVFs is not fully understood. However, accurate preangiographic detection of the location of the feeders is associated with a reduction of approximately half of the following fluoroscopy time. ${ }^{7}$ Therefore, $\mathrm{R}-\mathrm{CTA}$ is also expected to reduce the radiation dose of the following DSA. Because accurate detection of feeders of SAVFs is also possible, use of R-CTA should be considered. Further clinical studies are required to confirm this recommendation. Third, a relatively long postprocessing time is required. More than 4 hours accurately subtract bones. As a result, accurate diagnosis of feeders of SAVFs was achieved, despite the shorter interpretation time than conventional CTA.

In our study, we proposed weighted subtraction to invert bone attenuation to easily diagnose the level of the intervertebral foramen (Fig 3). In the usual bone-subtraction method, the background is totally equaled. Therefore, once optimal subtraction is performed, the landmark has disappeared. This feature makes it difficult to determine the level of the vertebra, which is the landmark for determination of the level of the segmental artery by using subtraction images alone. However, our proposed weighted subtraction provided an inverted shadow of the bones on the subtracted CTA images. The bone is displayed as a darker struc- 
ture than the background attenuation (Fig 3). As a result, the time for detecting feeders was reduced because the vertebral body or the rib bone could be recognized on the subtracted CTA images. Moreover, because the inverted bone shadow did not affect the visibility of vessels in MIP images, R-CTA provided clear, thinslab MIP images. Despite these advantages, this method slightly lowers the contrast of enhanced vessels compared with the usual bone-subtraction method. In addition, the optimization of the weighted value should be further investigated.

Our study has several limitations. First, the DSA results might not be independent from prior CTA results. However, routine DSA for the entire segmental artery in every patient is unacceptable because of its invasiveness. Second, R-CTA was generated by subtracting late arterial phase CTA. Noncontrast CT is theoretically suitable for subtraction because of the high difference in attenuation of vessels between contrast and noncontrast CT. However, we did not perform noncontrast CT with the same settings as spinal CTA. Finally, the population was small, and the study design was retrospective. Further larger and multicenter studies are required to clarify the feasibility of our proposed subtraction method in the clinical setting.

\section{CONCLUSIONS}

Bone-subtracted spinal CT angiography imaging by using novel nonrigid registration helps radiologists assess feeders of SAVFs more accurately and quickly than the conventional method.

\section{ACKNOWLEDGMENTS}

We are grateful to Dr Kensuke Uotani for his contribution of establishing the spinal CTA acquisition sequence. We also thank $\mathrm{Mr}$ Hajime Yamakage for assistance with statistical analysis in this study.

Disclosures: Mizuho Nishio_UNRELATED: Grants/Grants Pending: Toshiba, ${ }^{*}$ Comments: The grant was for $\mathrm{CT}$ research; Patents (planned, pending or issued): submission of patents with Toshiba, ${ }^{\star}$ Comments: The patent is related to computer-aided diagnosis. Eiji Kohmura—UNRELATED: Grants/Grants Pending: Ministry of Education, Culture and Science, ${ }^{\star}$ Comments: mainly on basic research on malignant brain tumor; Payment for Lectures (including service on Speakers Bureaus): Medtronic Japan; Royalties: microsurgical dissector development (Muranaka Medical Instruments Co., Ltd.). *Money paid to the institution.

\section{REFERENCES}

1. Krings T, Geibprasert S. Spinal dural arteriovenous fistulas. $A J N R$ Am J Neuroradiol 2009;30:639-48 CrossRef Medline

2. Ofran Y, Yovchev I, Hiller N, et al. Correlation between time to diagnosis and rehabilitation outcomes in patients with spinal dural arteriovenous fistula. J Spinal Cord Med 2013;36:200-06 CrossRef Medline

3. Zampakis P, Santosh C, Taylor W, et al. The role of non-invasive computed tomography in patients with suspected dural fistulas with spinal drainage. Neurosurgery 2006;58:686-94; discussion 686-94 Medline

4. Lai PH, Weng MJ, Lee KW, et al. Multidetector CT angiography in diagnosing type I and type IVA spinal vascular malformations. AJNR Am J Neuroradiol 2006;27:813-17 Medline

5. Yamaguchi S, Nagayama T, Eguchi K, et al. Accuracy and pitfalls of multi- detector-row computed tomography in detecting spinal dural arteriovenous fistulas. J Neurosurg Spine 2010;12:243-48 CrossRef Medline

6. Oda S, Utsunomiya D, Hirai T, et al. Comparison of dynamic contrast-enhanced 3T MR and 64-row multidetector CT angiography for the localization of spinal dural arteriovenous fistulas. AJNR Am J Neuroradiol 2014;35:407-12 CrossRef Medline

7. Luetmer PH, Lane JI, Gilbertson JR, et al. Preangiographic evaluation of spinal dural arteriovenous fistulas with elliptic centric contrast-enhanced MR angiography and effect on radiation dose and volume of iodinated contrast material. AJNR Am J Neuroradiol 2005;26:711-18 Medline

8. Takase K, Akasaka J, Sawamura Y, et al. Preoperative MDCT evaluation of the artery of Adamkiewicz and its origin. J Comput Assist Tomogr 2006;30:716-22 CrossRef Medline

9. Yoshioka K, Niinuma H, Ehara S, et al. MR angiography and CT angiography of the artery of Adamkiewicz: state of the art. Radiographics 2006;26(suppl 1):S63-73 CrossRef Medline

10. Jayakrishnan VK, White PM, Aitken D, et al. Subtraction helical CT angiography of intra- and extracranial vessels: technical considerations and preliminary experience. AJNR Am J Neuroradiol 2003;24: 451-55 Medline

11. Tomandl BF, Hammen T, Klotz E, et al. Bone-subtraction CT angiography for the evaluation of intracranial aneurysms. AJNR AmJ Neuroradiol 2006;27:55-59 Medline

12. Lell MM, Ditt H, Panknin C, et al. Bone-subtraction CT angiography: evaluation of two different fully automated imageregistration procedures for interscan motion compensation. AJNR Am J Neuroradiol 2007;28:1362-68 CrossRef Medline

13. Fujiwara H, Momoshima S, Akiyama T, et al. Whole-brain CT digital subtraction angiography of cerebral dural arteriovenous fistula using 320-detector row CT. Neuroradiology 2013;55:837-43 CrossRef Medline

14. Crum WR, Hartkens T, Hill DL. Non-rigid image registration: theory and practice. Br J Radiol 2004;77(Spec No 2):S140-53 CrossRef Medline

15. Avants BB, Epstein CL, Grossman M, et al. Symmetric diffeomorphic image registration with cross-correlation: evaluating automated labeling of elderly and neurodegenerative brain. Med Image Anal 2008;12:26-41 CrossRef Medline

16. Klein A, Andersson J, Ardekani BA, et al. Evaluation of 14 nonlinear deformation algorithms applied to human brain MRI registration. Neuroimage 2009;46:786-802 CrossRef Medline

17. Nishii T, Kono AK, Negi N, et al. The feasibility of a 64-slice MDCT for detection of the Adamkiewicz artery: comparison of the detection rate of intravenous injection CT angiography using a 64-slice MDCT versus intra-arterial and intravenous injection CT angiography using a 16-slice MDCT. Int J Cardiovasc Imaging 2013; 29(suppl 2):127-33 CrossRef Medline

18. Tanaka R, Yoshioka K, Muranaka K, et al. Improved evaluation of calcified segments on coronary CT angiography: a feasibility study of coronary calcium subtraction. Int J Cardiovasc Imaging 2013; 29(suppl 2):75-81 CrossRef Medline

19. Venema HW, Hulsmans FJ, den Heeten GJ. CT angiography of the circle of Willis and intracranial internal carotid arteries: maximum intensity projection with matched mask bone elimination-feasibility study. Radiology 2001;218:893-98 CrossRef Medline

20. Uotani K, Yamada N, Kono AK, et al. Preoperative visualization of the artery of Adamkiewicz by intra-arterial CT angiography. AJNR Am J Neuroradiol 2008;29:314-18 CrossRef Medline

21. Xia W, Gao X. A fast deformable registration method for 4D lung CT in hybrid framework. Int J Comput Assist Radiol Surg 2014;9: 523-33 CrossRef Medline 